Cahiers de recherches médiévales

\title{
La bibliothèque de Saint-Victor au service des rénovateurs de l'histoire de France vers 1500 ?
}

Nicole Gilles, Robert Gaguin et Paul Émile face aux ressources victorines

\section{Franck Collard}

\section{OpenEdition}

\section{Journals}

Édition électronique

URL : https://journals.openedition.org/crm/11524

DOI : $10.4000 / \mathrm{crm} .11524$

ISSN : 1955-2424

\section{Éditeur}

Honoré Champion

Édition imprimée

Date de publication : 15 juin 2009

Pagination : 227-240

ISSN : 1272-9752

\section{Référence électronique}

Franck Collard, «La bibliothèque de Saint-Victor au service des rénovateurs de l'histoire de France vers 1500 ? ", Cahiers de recherches médiévales [En ligne], 17 | 2009, mis en ligne le 15 juin 2012, consulté le 15 décembre 2022. URL : http://journals.openedition.org/crm/11524 ; DOI : https:// doi.org/10.4000/crm.11524 


\title{
rin
}

\section{La bibliothèque de Saint-Victor au service des rénovateurs de l'histoire de France vers 1500 ? Nicole Gilles, Robert Gaguin et Paul Émile face aux ressources victorines}

\begin{abstract}
Enjoying enormous prestige from the $12^{\text {th }}$ century on and open to humanist currents, Saint-Victor nonetheless represented a culture that was called into question by the Renaissance. How useful was its library, then, to the so-called renovators of the History of France that Nicole Gilles, Robert Gaguin and Paul Émile of Verona aspired to be around 1500 ? Despite the richness of Saint-Victor's relatively available resources, it seems that they were hardly used by the royal notary and secretary, or by the Italian humanist whose cultural choices were different. Only the Trinitarian and university doctor Robert Gaguin used, albeit sparingly, historical manuscipts housed at Saint-Victor, as a precise example of borrowing allows us to establish.
\end{abstract}

Résumé : Auréolée du prestige acquis depuis le XII siècle, mais symbole d'une culture remise en cause par la Renaissance, quoique ouverte à l'humanisme, la bibliothèque de Saint-Victor servit-elle aux rénovateurs de l'Histoire de France que se voulaient être Nicole Gilles, Robert Gaguin et Paul Émile de Vérone vers 1500 ? Malgré la richesse de ses ressources et leur relative accessibilité, elle ne semble guère avoir été mise à contribution par le notaire et secrétaire du roi comme par l'humaniste italien, aux horizons culturels autres. Seul le Trinitaire et universitaire Gaguin a recouru, et encore très modérément, aux volumes historiques victorins comme un exemple d'emprunt précis permet de l'établir.

Le nouvel âge ouvert par l'invention puis l'essor de l'imprimé a été, comme chacun sait, une période de profond renouvellement des conditions de documentation et de travail des historiens, ainsi qu'un temps d'intense renouvellement des textes de référence ${ }^{1}$. Dans cette époque de mutations marquée, chez les humanistes les plus en pointe ou les plus alignés sur les idées italiennes, par un rejet méprisant de la culture traditionnelle, les bibliothèques conventuelles passaient pour le cimetière des vieux savoirs périmés. Autrefois célébrée, la quantité de leurs lourds volumes désormais jugés illisibles autant par leur écriture que par leur style et par leur propos, était devenue un sujet de raillerie. La bibliothèque de Saint-Victor, constituée par quatre siècles d'acquisition et de copie, n'a pas échappé à ce destin. Elle était tenue par le Padouan Scaliger (1484-1558) pour ne rien posséder qui vaille ${ }^{2}$. Elle fut prise pour cible par Rabelais dans son Pantagruel (1532). En fabriquant un inventaire parodique «des beaux livres de la librairie de

${ }^{1}$ Voir E. Eisenstein, La révolution de l'imprimé à l'aube de l'Europe moderne, Paris, Hachette (tr. fçse), 2003.

${ }^{2}$ G. Ouy, Les manuscrits de l'abbaye de Saint-Victor, catalogue établi sur la base du répertoire de Claude de Grandrue, Turnhout, Brepols, 2 vol., 1999 (Bibliotheca victorina X), p. 60.

Cahiers de Recherches Médiévales, 17, 2009 
Saint-Victor », il la tourna en dérision et, à travers elle, la culture scolastique devenue totalement inadaptée aux temps nouveaux ${ }^{3}$.

Pour que la charge fût forte, Rabelais avait choisi un haut lieu du savoir, longtemps renommé pour son prestige intellectuel et sa richesse livresque. De son temps, tout le monde ne partageait pas le sentiment caricatural de l'écrivain. Sans parler des Victorins eux-mêmes, évidemment fiers d'une bibliothèque bien vivante dont la reconstruction matérielle ${ }^{4}$ et le nouveau catalogage étaient entrepris dans les années 1508-1514, il était des gens pour apprécier les ressources offertes par l'abbaye. Lors d'un procès de 1561 mettant en jeu la limite géographique de souveraineté du roi de France, un avocat mentionna «plusieurs vieilles et anciennes croniques escriptes à la main [que nous avons] trouvées en la librairie St Victor... ». Elles venaient compléter, disait-il, les ouvrages abondamment sollicités de Robert Gaguin $(† 1501)$, Nicole Gilles $(† 1503)$ et Paul Émile $(\dagger 1529)^{5}$.

Prenant le relais de la production de Saint-Denis, jugée inélégante et fautive ${ }^{6}$, ces rénovateurs de l'histoire de France publièrent leurs œuvres à peu près au même moment ${ }^{7}$. Deux se réclamaient de l'humanisme, sans toutefois partager la même vision de la culture traditionnelle, qu'en bon Italien le Véronais Paul Émile rejetait plus franchement que le régnicole Gaguin ${ }^{8}$; le troisième, écrivant en langue vulgaire, illustrait la tradition historiographique des notaires et secrétaires du roi

\footnotetext{
${ }^{3}$ Rabelais, Pantagruel, ch. $7:$ : Comment Pantagruel vint à Paris et des beaux livres de la librairie de Saint-Victor».

${ }^{4}$ L. Delisle, Le cabinet des manuscrits de la Bibliothèque Nationale, t. 2, Paris, 1874, p. 200, 226, 254, signale la vague d'édification de nouvelles bibliothèques à la Sorbonne (1481), au collège de Navarre sous Charles VIII, aux Mathurins de Paris (1485) et à Saint-Victor, presque entièrement reconstruit entre 1500 et 1525 . J.-M. Le Gall, Les moines au temps des réformes (1480-1560), Seyssel, Champ-Vallon, 2001, p. 193 et sq. note cependant que les établissements réformés ne sont pas à la pointe d'un mouvement trop favorable à la culture «mondaine ».

${ }^{5}$ Cité par B. Guenée, «Des limites féodales aux frontières politiques », Les lieux de mémoire, dir. P. Nora, t. 2, La Nation, vol. 2, Le territoire, Paris, 1986, p. 11-33, p. 26.

${ }^{6}$ Nicole Gilles, Annales de France, éd. de Paris, 1525, parle de la «multitude et confusion des matieres » (f. $\left.1^{\mathrm{v}}\right)$. Le point sur cette question est fait dans F. Collard, Un historien au travail à la fin du XV siècle : Robert Gaguin, Genève, Droz, 1996, p. 85-98. Nous nous permettons d'y renvoyer le lecteur.

${ }^{7}$ Robert Gaguin, Compendium de origine et gestis Francorum, $1^{\text {ère }}$ éd. Paris, 1495 ; les références renverront toujours à l'édition de Paris, 1500/1501, la dernière parue du vivant de

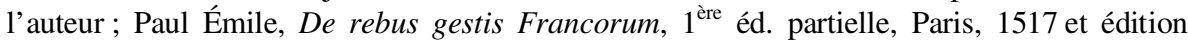
intégrale Paris, 1539 ; l'œuvre de Nicole Gilles connut sans doute des éditions antérieures à 1525 mais elles sont perdues.

8 Sur ces deux auteurs, voir principalement K. Davies, Late $X V^{\text {th }}$ Century French Historiography as exemplified in the "Compendium" of Robert Gaguin and the "De rebus gestis" of Paulus Aemilius, Edimbourg, 1954 ; S. Charrier, Recherches sur l'œuvre latine en prose de Robert Gaguin, Paris Champion, 1996 ; F. Collard, Un historien au travail...; Th. Maissen, Von der Legende zum Modell. Das Interesse an Frankreichs Vergangenheit während der italienischen Renaissance, Bâle et Francfort-sur-le-Main, Helbing \& Lichtenhahn, 1994, p. 176-210.
} 
mais comptait la ressourcer". Tous devaient nourrir leur propos de sources multiples. Pour autant qu' on les connaisse ${ }^{10}$, leurs bibliothèques personnelles ne suffisaient pas à fournir les «multiples volumes » à consulter ${ }^{11}$. Profitèrent-ils des ressources de Saint-Victor, eux qui résidèrent plus ou moins continûment à Paris, ou les dédaignèrent-ils comme dépassées ou inadéquates à leur entreprise ? Telle est la question à trancher.

Emprunter des livres ou en consulter sur place pour en extraire des matériaux historiques relèvent du travail ordinaire de l'historien vers $1500^{12}$. La difficulté est que les auteurs n'en font guère mention dans leur texte, ou alors très allusivement, moins par ingratitude que par volonté de présenter un «produit fini » qui laisse de côté, au profit de la fluidité du récit, les circonstances de sa confection, à moins que celles-ci ne donnent du poids au récit. Faute de la subsistance de registres de prêt (sauf cas d'exception ${ }^{13}$ ), ce sont plutôt les plaintes des bibliothécaires devant la disparition des ouvrages qui nous renseignent sur leur utilisation, mais sans indiquer leurs utilisateurs. À Paris et dans les environs, des «librairies» conventuelles permettaient à des gens extérieurs munis de bonnes raisons de profiter de leurs fonds. Ainsi en allait-il à Saint-Germain-des-Près dès le XIII ${ }^{\mathrm{e}}$ siècle. Un auteur écrivant au début du règne de Charles VIII affirme y avoir consulté un livre de chroniques $^{14}$. Même possibilité à Saint-Denis ${ }^{15}$. Il en allait très probablement de

\footnotetext{
${ }^{9}$ Se reporter aux travaux de K. Daly sur la production historiographique des notaires et secrétaires du roi, et notamment à l'introduction de sa récente édition de l'Abrégé des croniques de France de Noël de Fribois, Paris, Champion, 2006. Sur Nicole Gilles précisément, nulle étude d'ensemble n'a été faite depuis 1930 (J. Riche, «L'historien Nicole Gilles (14?-1503)», Positions de thèses de l'École des Chartes, 1930, p. 135-142).

${ }^{10}$ Celle de Paul Émile est inconnue ; celle de Gaguin était réputée pour sa richesse que vante Érasme (Epistole, éd. P. S. Allen, Oxford, 1906, n 121, t. 1 p. 283) mais il n’en subsiste hélas que des inventaires dressés au XVIII ${ }^{\mathrm{e}}$ siècle après de considérables pertes dues à un ministre général indélicat (F. Collard, Un historien au travail..., p. 98-100); celle de Nicole Gilles a été inventoriée en 1499 à l'occasion de la mort de sa femme et son inventaire figure dans R. Doucet, Les bibliothèques parisiennes au XV siècle, Paris, 1956, p. 83-89.

${ }^{11}$ Paul Émile, De rebus gestis Francorum, édition de 1539, prologue.

${ }^{12}$ B. Guenée, Histoire et culture historique, p. 109-12. En 1498, l'historien breton Pierre le Baud se fit ainsi ouvrir une vingtaine de bibliothèques du duché de Bretagne avec l'appui de la duchesse Anne.

${ }^{13} \mathrm{~J}$. Vielliard, «Le registre de prêt de la bibliothèque du collège de Sorbonne au $X \mathrm{~V}^{\mathrm{e}}$ siècle », The Universities in the Late Middle Ages, éd. J. Ijsewijn et J. Paquet, Louvain, 1978, p. 276293, signale, p. 291, l'absence d'emprunts de livres d'histoire : est-ce-à-dire qu'il s'en trouvait peu?

${ }^{14}$ L. Delisle, Le cabinet des manuscrits, t. 2 p. 42.

${ }^{15}$ D. Nebbiai Dalla Guarda, La bibliothèque de l'abbaye de Saint-Denis en France du IX $X^{e}$ au XVIII siècle, Paris, 1985, p. 90, donne le seul exemple connu mais significatif de Jean de Berry empruntant en 1415 un exemplaire de la chronique latine de Guillaume de Nangis qu'il voulait faire copier.
} 
même à Saint-Victor, malgré l'instauration de règles d'usage plus strictes en 1392 afin d'éviter les vols ${ }^{16}$.

L'histoire de l'abbaye et de sa bibliothèque, entreprise dès le XVII ${ }^{\mathrm{e}}$ siècle par Jean de Thoulouze, n'a pas à être refaite ici $^{17}$. Ce sont seulement la consistance et le renom de cette dernière vers 1500 qui doivent être rappelés. Le renom était grand. Quoique bien moins ancienne que d'autres abbayes puisque fondée en 1113, SaintVictor passait pour posséder un fonds de livres considérable. Un texte intitulé Corona duodecim stellarum domus Sancti Victoris Parisiensis et dû, dans la seconde moitié du XV $\mathrm{XV}^{\mathrm{e}}$ siècle, à Jean Mauburnus (Mombaer) de Bruxelles (†1502), envoyé en mission réformatrice dans le diocèse de Paris, estime que la dixième étoile de l'abbaye est sa famosissima libraria ${ }^{18}$. Venu avec lui en octobre 1497, le chanoine de Windesheim Corneille Gérard de Gouda, fin connaisseur des livres puisque bibliothécaire du couvent de Hemsdonck, au diocèse de Dordrecht, en souligne plus particulièrement la richesse en œuvres historiques: Et legere historias huc sine fraude veni écrit-il en un vers ${ }^{19}$.

La meilleure preuve qu'il ne s'agit pas de flatteries de circonstances réside dans le fameux catalogue réalisé en 1513-1514 par Claude de Grandrue, bibliothécaire du lieu. Sa confection est consécutive, sans doute, à la reconstruction de l'abbaye où une vaste salle de lecture percée de nombreuses fenêtres fut aménagée en 1508 au plus $\operatorname{tar}^{20}$. Le catalogue comptait 1081 entrées. Claude de Grandrue déplorait la disparition de 25 volumes parmi lesquels, apparemment, aucun ouvrage d'histoire, mais des lettres de Pline dérobées par un Dominicain italien indélicat, preuve que les fonds pouvaient intéresser des humanistes ${ }^{21}$ (il contenait aussi la Guerre gothique de Procope traduite par Bruni ${ }^{22}$ ) et conséquence fâcheuse d'une trop généreuse ouverture aux étrangers, malgré le statut sévère de $1392^{23}$.

\footnotetext{
${ }^{16}$ Voir A. Franklin, Histoire de la bibliothèque de l'abbaye de Saint-Victor à Paris d'après des documents inédits, Paris, 1865, p. 36.

${ }^{17}$ Voir l'Histoire des bibliothèques françaises, I, Les bibliothèques médiévales du VI ${ }^{e}$ s. à 1530, Paris, Promodis, 1989, p. 81 et sq. et G. Ouy, Les manuscrits de l'abbaye de SaintVictor, p. 15sq. ; L. Delisle, Le cabinet des manuscrits, t. 2 p. 209-235. La majeure partie de ce qui suit en provient.

${ }^{18}$ Recueil du XVII ${ }^{\mathrm{e}}$ siècle consacré à Saint-Victor, ms. lat. 14366 de la BNF, f. 45-46.

${ }^{19} \mathrm{Ibid}$. Il poursuit en disant que tout ce que la pensée a produit se trouve à la Saint-Victor : Platon, Aristote, Apulée, Ptolémée, du droit canon...

${ }^{20}$ A. Franklin, Histoire de la bibliothèque de l'abbaye de Saint-Victor, p. 28.

${ }^{21}$ La bibliothèque possédait des classiques latins notamment grâce aux apports du premier humanisme français. Voir l'Histoire des bibliothèques françaises, I, p. 86 et 276 et G. Ouy, Les manuscrits de l'abbaye de Saint-Victor, p. 15 et sq. Très tôt, elle accueillit des imprimés, le premier étant l'édition parisienne (1471) des lettres de saint Jérôme.

${ }^{22}$ Actuels mss. lat. 15070 et 15071.

${ }^{23}$ G. Ouy, Les manuscrits de l'abbaye de Saint-Victor, p. 26 et sq., estime que l'ère de croissance accélérée de la bibliothèque, dans les années 1420-1450, au temps de Jean Lamasse, prieur (1422) puis abbé (1448), fut suivie par une période moins florissante car marquée de nombreuses pertes intervenues entre 1450 et 1514 . Les donateurs étaient inscrits à
} 
La bibliothèque était ouverte aux étudiants de droit et de médecine car elle possèdait de beaux fonds en ces disciplines. Comme le suggérait Corneille Girard, l'histoire n'était pas en reste. Cotés au moyen d'une triple lettre (représentant un des 52 pupîtres où étaient les volumes) suivie d'un numéro, les ouvrages historiques étaient au nombre de plusieurs dizaines, le plus souvent rassemblés dans des recueils composites. Par chance subsistent une très grande partie de ces volumes, versés très majoritairement en 1796 à la Bibliothèque nationale. Les travaux de G. Ouy et de V. Gerz von Büren permettent de les bien connaître ${ }^{24}$. Leur énumération exhaustive n'aurait guère d'intérêt. Retenons simplement quelques manuscrits susceptibles de rendre des services à nos trois historiens de la France.

L'histoire nationale était représentée par quelques textes généralistes ou plus limités dans le temps. Grand classique, le De gestis Francorum d'Aimoin de Fleury, avec continuation, offrait de vastes ressources (actuel ms. lat. 15046 de la BNF), de même que l'Historia Francorum d'Hugues de Fleury (actuel ms. lat. 15047 de la $\mathrm{BNF}$ ). Ce qui regarde la Gaule était accessible dans des ouvrages antiques couramment exploités par les historiens médiévaux comme Eutrope et Justin (actuels mss. lat. 14693 et 14623 de la BNF). La Vita Karoli d'Éginhard était sur les pupîtres (actuel ms. lat. 14663 de la BNF) de même que le Liber Turpini de historia Karoli magni (actuel ms. lat. 14703 de la BNF).

Qui recherchait des éléments sur l'histoire particulière des principautés constituant le royaume ou des dominations voisines pouvait également trouver de quoi satisfaire sa curiosité. Guillaume de Jumièges, historien des ducs de Normandie, figurait par exemple dans divers manuscrits (actuels mss. lat. 14663 et 15047 de la BNF). L'Historia Longobardorum de Paul Diacre était à disposition (actuel ms. lat. 14693 de la BNF). De même, l'histoire des croisades était bien présente ${ }^{25}$.

Mais c'était, assez logiquement pour une bibliothèque monastique, l'histoire universelle qui était la mieux représentée au travers d'œuvres du reste parfaitement utilisables à des fins d'écriture de l'histoire du royaume en raison de leur fréquente orientation française ${ }^{26}$ et de leur précision chronologique. Citons la chronique de Sigebert de Gembloux (actuel ms. lat. 14624 de la BNF), la Martiniane (actuel ms. lat. 14357) et le Memoriale historiarum de Jean de Saint-Victor, inévitablement présent sous différentes formes (fragmentaires, continuées) dans les fonds de l'abbaye (actuel mss. lat. 14626, 15010, 15011 de la BNF), comme d'ailleurs la chronique attribuée à Hugues de Saint-Victor, très utile pour le comput (actuels mss.

l'obituaire de l'abbaye dont ils bénéficiaient des prières. Leur liste a été conservée par exemple dans le ms. lat. 14677 de la BNF, f. $378^{\mathrm{v}}-380$.

${ }^{24}$ Le catalogue de la bibliothèque de l'Abbaye de Saint-Victor de Paris de Claude de Grandrue, 1514, éd. V. Gerz von Büren et G. Ouy, Paris, éd. du CNRS, 1983.

${ }^{25}$ Exemple Liber historiarum partium Orientis, d'Hayton, traduit en latin en 1307 (actuel ms. lat. 14693 de la BNF); Jacques de Vitry, Historia orientalis et occidentalis (actuel ms. lat. 14436 de la BNF) ; la chronique de Foucher de Chartres sur la première croisade (actuel ms. lat. 15038 de la BNF) ou celle de Baudri de Bourgueil, actuel ms. lat. 15075 de la BNF.

${ }^{26}$ Sur cette question, voir l'ouvrage fondamental de Mireille Chazan, L'Empire et l'histoire universelle de Sigebert de Gembloux à Jean de Saint-Victor (XII ${ }^{e}$-XIV ${ }^{e}$ siècle), Paris, Champion, 1999. 
lat. 14872 et 15009$)^{27}$. Deux autres ouvrages, des plus lus, étaient aussi à la disposition des lecteurs : la chronique latine de Guillaume de Nangis, si riche en données sur l'histoire de France (actuel ms. lat. 14358 de la BNF), et le Speculum historiale de Vincent de Beauvais doté, dans le manuscrit victorin, des précieuses tables de Jean Hautfuney permettant un usage ciblé de l'ouvrage (actuels mss. lat. 14354 à 14356 de la BNF).

Un manuscrit rassemblait tous ces genres à la fois, avec, en sus, des textes de nature juridique, diplomatique et politique. Il pouvait constituer une vraie mine pour un historien de la France. C'est le manuscrit actuellement côté lat. 14663 de la BNF. G. Ouy en a décrit l'histoire ${ }^{28}$. Résumons-la. Admis comme boursier à l'abbaye qui servait de collège aux chanoines réguliers de Saint-Augustin ${ }^{29}$, licencié en décret, le Normand Simon de Plumetot, devenu membre du Parlement de Paris et favorable au régime lancastrien, aimait les livres (en particulier autographes) et les humanités. Faute de réintégration par Charles VII dans la cour parisienne, il rejoignit l'Échiquier de Normandie et mourut à Rouen en 1443, riche de nombreuses prébendes. C'est au moment de quitter la capitale reconquise par le Valois en 1436 qu'il confia par prudence ses manuscrits à Saint-Victor dont le prieur Lamasse s'empressa de les intégrer au fonds de la librairie en faisant méthodiquement disparaître la marque de leur provenance ${ }^{30}$ et inscrire le nom du nouveau propriétaire $^{31}$. Parmi les 74 volumes identifiés comme venant de Simon de Plumetot se trouve ce recueil copié par ses divers copistes attitrés ou par lui-même et coté BBB4 par Claude de Grandrue. Parvenu amputé de presque 150 feuillets (fol. anciennement numérotés 251 à 396), mais doté (peut-être postérieurement à sa confection) d'une commode table des matières, il reflète les intérêts de son possesseur pour l'histoire et les privilèges de la monarchie ainsi que le droit et les rapports franco-anglais avec leurs points d'achoppement: Normandie, Ponthieu, Anjou $^{32}$.

\footnotetext{
${ }^{27}$ La notice du Dictionnaire des lettres françaises. Le Moyen Âge, consacrée à Hugues de Saint-Victor tient pour authentique cette chronique. Le catalogue de Claude de Grandrue, qui la désigne sous les cotes HH 3 (f. 51-86) et HH 6 (f. 3-42) la tient en tout cas pour telle. L'abrégé qui suit le texte du ms. lat. 15009 n'est pas d'Hugues en revanche.

${ }^{28}$ G. Ouy, Les manuscrits de l'abbaye de Saint-Victor, p. 15.

${ }^{29}$ Histoire des bibliothèques françaises, I, p. 85.

${ }^{30}$ G. Ouy, «Historique de la Bibliothèque de Saint-Victor », Le catalogue de la bibliothèque de l'abbaye de Saint-Victor, éd. V. Gerz von Büren, p. X-XII. Texte repris dans G. Ouy, Les manuscrits de l'abbaye de Saint-Victor, op. cit.

${ }_{31} \mathrm{Au}$ bas du premier feuillet de la première œuvre, la mention sans doute postérieure à la copie : hic liber est sancti victoris parisiensis. Inveniens quis ei reddat amore dei.

${ }^{32}$ Voici le contenu du recueil, écrit tout en latin sauf exception signalée: des tables astronomiques en page de garde et table des matières en face du premier feuillet numéroté ; 1Origine des comtes d'Anjou; 2-Succession des papes aux $\mathrm{XIV}^{\mathrm{e}}$ et $\mathrm{XV}^{\mathrm{e}}$ siècles ; 3-Lois de Lycurgue ; 4-Gestes des rois de France en français jusqu'à Philippe de Valois et continuation ; 5-Catalogues historiques des archevêques de Sens et de Rouen, des évêques d'Auxerre ; 6Abrégé d'histoire sainte; 7-Fragments concernant Paris du prologue donné par Raoul de Presles à sa traduction française de la Cité de Dieu de saint Augustin ; 8-Poème sur l'origine des lys ; 9-Valeur numérale des lettres ; 10-Catalogue des ordres religieux ; 11-Fragments
} 
Les fonds historiques de la librairie de Saint-Victor étaient donc assurément assez riches et probablement assez accessibles pour avoir pu attirer des historiens. Ces derniers pouvaient même y trouver des pièces d'archives concernant les deux procès de Jeanne d'Arc ${ }^{33}$. Toutes ces ressources en puissance sont-elles devenues des sources effectives?

Pour répondre, il convient d'abord de prendre en compte le profil et le projet historiographique des auteurs retenus. Ce dernier, on l'a dit, consistait dans les trois cas à écrire une histoire nationale. Qu'elle fût en latin ou en français, celle-ci devait se nourrir de sources - latines surtout et vulgaires un peu - allant bien au-delà des Historiae ou autres Gesta Francorum antérieures. En la matière, on l'a vu, l'offre victorine était de qualité. Mais le profil des auteurs s'adaptait plus ou moins bien à la fréquentation de la librairie de l'abbaye.

Le moins susceptible a priori de l'utiliser était Nicole Gilles ${ }^{34}$. Certes, il mentionne dans son œuvre la fondation de l'abbaye par Louis VI: «en recongnoissance de la victoire qu'il avoit obtenue contre ses ennemys, en l'honneur de monseigneur sainct Victor auquel il avoit singuliere devotion, il fonda et feit aedifier l'Eglise et Abbaye de sainct Victor lez Paris et y meit religieux de l'ordre de sainct Augustin $»^{35}$. Et il salue Hugues de Saint-Victor comme il se doit ${ }^{36}$. Mais,

concernant la dignité et les privilèges de la couronne de France copiés du prologue donné par Raoul de Presles à sa traduction française de la Cité de Dieu de saint Augustin; 12Généalogie des rois de France pour montrer leurs droits à la couronne de Navarre, par Richard Scot (Lescot ?) ; 13-Origines du royaume d'Yvetot ; 14-Extraits de la Guerre des Gaules de César sur les mœurs des Germains et des Gaulois ; 15-Extrait de Landolfo de Colonna sur la translation de l'Empire romain; 16-Vita Karoli secundum Guertinum ; 17-Histoire des ducs de Normandie par Guillaume de Jumièges ; 18-Chronique de Robert du Mont (de Thorigny) allant de 1139 à 1168 ; 19-Continuation de la précédente jusqu'en $1272 ; 20$-Chronique de Normandie de 1087 à 1239; 21-Épitaphes de Richard Cœur-de-Lion, Rollon, et vers sur Gautier de Coutance, archevêque de Rouen ; 22-Liste des comtes de Toulouse ; 23-Traité passé entre saint Louis et Henri III d'Angleterre ; 24-Gestes abrégés des rois de France jusqu'en 1137 ; 25-Sur les maires du Palais ; 26-Coutumes d'Angleterre ; 27-Historia regum Francorum jusqu'à Philippe Auguste ; 28-Hommes illustres ; 29-Chroniques abrégées en français attribuées à Guillaume de Nangis ; 30-Extrait d'une chronique de Saint-Wandrille ; 31-Noms des rois de France; 32-Sur le comté de Ponthieu; 33-Histoire de Nithard ; 34Annales de Flodoard.

${ }^{33} \mathrm{BNF}$, ms. lat. 14665, recueil sur les actes et le procès de Jeanne d'Arc copié vers 1500 à la demande de l'abbé Nicaise de l'Orme, comme c'est indiqué au bas du f. $2^{\mathrm{v}}$. Ph. Contamine, «Naissance d'une historiographie. Le souvenir de Jeanne d'Arc en France et hors de France depuis le "procès de son innocence" (1455-1456) jusqu'au début du XVI ${ }^{\mathrm{e}}$ siècle », Francia, 15 (1987), p. 233-56.

${ }^{34} \mathrm{Au}$ temps du premier humanisme français animé notamment par des notaires et secrétaires du roi, la bibliothèque victorine avait cependant tenu un certain rôle. Elle fut par ailleurs lieu de dépôt de livres reçus par le monarque.

${ }^{35}$ Chroniques et annales de France, Paris, éd. de 1566, f. $85^{\vee}$. 
représentant d'une historiographie laïque et administrative meilleure connaisseuse sans doute des textes gardés dans les archives ou dans la librairie royale que des manuscrits conservés dans les couvents, plus à l'aise avec le français qu'avec le latin, il ne paraît pas tourné vers les ressources victorines ${ }^{37}$.

Chanoine de Notre-Dame, mais plus clerc de cour que d'autel, l'Italien Paul Émile semble évoluer lui aussi dans un univers assez étranger au monde monastique et à sa culture, sans qu'il puisse être prouvé qu'il éprouvait déjà le mépris affiché plus tard par Rabelais, Érasme ou Scaliger. Il est seulement permis de constater que, contrairement aux œuvres de ses rivaux, son De rebus gestis Francorum ignore la fondation de l'abbaye dans le récit du règne de Louis VI. Par ailleurs, ses fonctions d'historiographe du roi (occupées au plus tard à partir de 1489) devaient lui ouvrir les portes des collections royales ${ }^{38}$. Certes, elles lui permettaient sans doute aussi d'avoir ses entrées dans les bibliothèques conventuelles, mais lui était-il vraiment nécessaire de fréquenter celle de Saint-Victor?

C'est à tous égards Robert Gaguin qui semble le plus proche de l'abbaye. Topographiquement parlant, son couvent des Mathurins n'était pas très éloigné de celui des Victorins. Spirituellement parlant, en sus du bon accueil que Saint-Victor semble avoir réservé aux processions des captifs libérés par l'Ordre de la Très Sainte Trinité $^{39}$, son ministre général, très ouvert aux courants de la Devotio moderna, suivait d'un œil favorable la tentative de réforme de l'établissement ${ }^{40}$ par des chanoines de la congrégation de Windesheim dont l'échec le consterna en $1497^{41}$. Culturellement parlant enfin, l'humaniste artésien était en phase avec Saint-Victor. Tenant le XII ${ }^{\mathrm{e}}$ siècle pour une grande époque du rayonnement intellectuel parisien et français $^{42}$ (ce que Paul Émile, adepte des schémas pétrarquiens, ne pensait en rien quant à lui), il marque son admiration à l'égard de l'établissement victorin en

\footnotetext{
${ }^{36}$ Ibid., f. $86^{\mathrm{v}}$ : « En ce temps fut Hugues de Saint Victor, tresexpert es sept ars liberaux, plus que nul autre, tellement qu'on disoit que c'estoit un autre sainct Augustin ; et feist le livre des sacremens qui estoit fort necessaire et plusieurs autres belles escriptures ».

${ }^{37} \mathrm{La}$ bibliothèque du notaire et secrétaire du roi renferme une écrasante majorité de livres en français.

${ }^{38}$ L. Delisle, «Documents parisiens de la bibliothèque de Berne », Mémoires de la société de l'histoire de Paris et de l'Île de France, 23 (1896), p. 224-98, donne l'exemple du manuscrit intitulé Origo Francorum seu chronicon Francorum ab anno Domini 380 usque ad 1308, conservé à Berne (ms. 70) et mis à la disposition de Nicole Gilles et de Paul Émile par le roi (p. 244)

${ }^{39}$ Antiquitatum abbatiae Sancti-Victoris libri XII, BNF ms. lat. 14677, f. 432, mention d'une procession organisée le 24 avril 1491 par les Trinitaires des Mathurins qui amenèrent sur le seuil de l'abbaye de Saint-Victor des captifs libérés de sorte que le peuple les voie; ils rentrent et sortent de l'église ; un sermon est fait à leur propos dans le cimetière des Victorins. ${ }^{40}$ Sur la question, voir J.-M. Le Gall, Les moines au temps des réformes (1480-1560), p. 40.

${ }^{41}$ Sur ses relations avec Corneille, voir Epistole et orationes, éd. L. Thuasne, Paris, 1903, n ${ }^{\circ}$ 89 bis, 1498, t. 2 p. 76. Sur la mission des réformateurs et son échec, voir les annales laissées par Pierre Boucher et insérées par Jean de Thoulouze dans les Antiquitatum abbatiae SanctiVictoris libri XII, BNF ms. lat. 14677, f. $425^{\mathrm{v}}-426$.

${ }^{42}$ F. Collard, Un historien au travail, p. 54 et sq., repris et mis à jour dans la notice «Robert Gaguin » de l'Histoire littéraire de la France, t. 43-1, Paris, 2005, p. 173-213.
} 
insérant dans son Compendium un passage sur la fondation de Louis VI et des vers la célébrant ${ }^{43}$ puis en mentionnant, quelques pages plus loin, la grande figure d'Hugues de Saint-Victor négligé dans les trois premières éditions ${ }^{44}$. Ajoutons qu'en tant que docteur régent de la faculté de décret, le maître de l'Université devait connaître les ressources de la librairie victorine en droit canon. Certes, Gaguin semble absent de l'annalistique victorine de la fin du $X V^{\mathrm{e}}$ siècle telle que Jean de Thoulouze l'a reproduite deux siècles plus tard. Mais bien des éléments autorisent à penser que l'auteur du Compendium avait des rapports étroits avec l'abbaye.

Toute la question est de prouver, pour lui comme pour ses collègues, un recours aux volumes de la bibliothèque de Saint-Victor. La chose est compliquée à maints égards. D'une part, nos auteurs ne disent rien ou disent peu de leurs conditions de documentation. Ils sont de surcroît loin de fournir la liste de leurs sources, alléguées simplement de temps à autres, et plus que rarement chez Paul Émile. D'autre part, les textes à partir desquels on sait ou on pense qu'ils ont travaillé sont très répandus. Il est par conséquent extrêmement difficile de décider de l'endroit où ils les ont consultés, fait copier ou empruntés, sauf à disposer de la connaissance de leur bibliothèque. Prenons l'exemple du Speculum historiale, reproduit en de nombreux autres manuscrits et existant aussi sous la forme imprimée qui eut les suffrages de Nicole Gilles, possesseur de l'incunable du Myroir historial en français. La chronique latine de Guillaume de Nangis se trouve pareillement dans maintes bibliothèques tout comme l'œuvre d'Aimoin, la Vita Karoli ${ }^{45}$ ou encore l'ouvrage de Guillaume de Jumièges et celui de Sigebert de Gembloux. Il faut se limiter à porter l'attention sur quelques textes ou recueils de textes moins courants ou plus spécifiquement liés à l'abbaye ${ }^{46}$.

Parmi eux, deux retiennent l'attention. Le premier est celui d'Hugues de Saint-Victor. Sa chronique était connue de Nicole Gilles qui la cite nommément, à propos de dates fixant la destruction de Troie, en compagnie de «celuy qui feist les

\footnotetext{
${ }^{43}$ Compendium, f. 48 : Huius ludovici... opus est divi victoris coenobium quod in suburbano parisiano ad orientem solem a fundamentis extruxit ; Illustris genitor ludovici rex ludovicus / Vir clemens christi servorum semper amicus / Institui fecit pastorem canonicorum / In cella veteri trans flumen parisiorum. / Hanc vir magnanimus almi victoris amore / Auro reliquiis ornavit rebus honore / Sancte dionysi qui servas corpus humatum / Martyr et antistes ludovici solve reatum.

${ }^{44}$ Ibid., f. $50^{v}$ : Hac etate claruit illustris doctor Hugo de sancto victore.

${ }^{45}$ Il est subsiste plus de 80 manuscrits selon l'éditeur-traducteur de la Vita Karoli, Louis Halphen, Paris, 1923, introduction.

${ }^{46}$ La bibliothèque comportait d'ailleurs deux pupîtres spécialement dédiés aux «auteurs victorins » cotés en $\mathrm{HH}$ et $\mathrm{JJ}$.
} 
Chroniques de France... $\gg^{47}$. Mais citer n'est pas toujours consulter et consulter pouvait se faire ailleurs qu'à Saint-Victor car le texte est sorti de son incubateur ${ }^{48}$.

Le second texte est celui de Jean de Saint-Victor. Il demeure une douzaine de manuscrits médiévaux de son ouvrage dont trois appartenant au fonds de l'abbaye. Il fut copié à au moins cinq reprises dans le seconde moitié du $X^{\mathrm{e}}$ siècle ${ }^{49}$. Nicole Gilles ne laissent pas voir qu'il se soit servi du Memoriale Historiarum. En revanche, il est assuré que Gaguin y eut recours pour quelques (courts) passages du Compendium. Il aurait plutôt utilisé le lat. 15011 que le lat. 14626 (pourtant considéré comme l'exemplaire mis à la disposition des lecteurs étrangers à l'abbaye par les moines $)^{50}$ à cause d'un texte en vers déjà mentionné sur le roi Louis VI, absent du second mais ajouté en marge du premier ${ }^{51}$. La formule de Gaguin, de quo sequens epigramma scriptum invenio pourrait évidemment laisser penser que le Trinitaire avait lu l'inscription sur les murs du cloître de l'abbaye où elle se trouvait effectivement. Ce qui détourne de le croire, c'est le caractère incomplet du texte donné par le Trinitaire, exactement tronqué comme celui du manuscrit ${ }^{52}$. D'autres

\footnotetext{
${ }^{47}$ Croniques et Annales de France, f. 7, an 3978 ou 3980 de la Création, 1190 av. J.C. Le problème est que dans les deux manuscrits victorins consultés, ces données ne se retrouvent pas. Mais Gilles ne s'est pas contenté de recopier les Grandes chroniques de France car leur récit est vierge de toute allusion à Hugues de Saint-Victor.

${ }^{48} \mathrm{La}$ BNF possède par exemple deux manuscrits étrangers au fonds de Saint-Victor. L'un appartenait à la riche bibliothèque de Saint-Gemain-des-Prés (BNF mss. lat. 4802 ou 13409). On trouve aussi des manuscrits à Troyes, Boulogne/Mer, Cambrai...

${ }^{49}$ I. Guyot-Bachy, Le Memoriale historiarum de Jean de Saint-Victor : un historien et sa communauté au début du XIV siècle, Turnhout, 2000, p. 22-47 et sq. Signalons, d'après cet ouvrage, un manuscrit incomplet et ayant appartenu aux Carmes de Paris, première version du texte, sans "postérité » codicologique (Ars. 1117); un autre copié en 1484 pour Hugues le Breton, lieutenant du bailli de Nevers (BNF ms. lat. 4941) ; un autre copié à et détenu par Sainte-Geneviève (ms. 516) mais de date incertaine (fin $\mathrm{XV}^{\mathrm{e}}$ ou début $\mathrm{XVI}^{\mathrm{e}}$ ); un autre disparu mais signalé début $\mathrm{XV}^{\mathrm{e}}$ comme appartenant à l'abbaye bénédictine de Saint-Magloire. ${ }^{50}$ I. Guyot-Bachy, Le Memoriale historiarum..., p. 28.

${ }^{51}$ Compendium, f. $48 \mathrm{v} /$ Memoriale historiarum, BNF ms. lat. 15011, f. 381 : huitain ajouté au bas du feuillet sur deux colonnes sous le titre epitafium ludivici grossi regis francorum et copiés maladroitement: la $6^{\text {ème }}$ ligne tout en bas de la feuille, est quasi illisible. Le texte, ajouté de la main de Jean de Saint-Victor, et strictement identique à celui que reproduit Gaguin, aurait été intégré, comme tous les ajouts portés dans les marges, aux copies postérieures (I. Guyot-Bachy, Le Memoriale historiarum..., p. 26). Il est pourtant absent, on l'a dit, du lat. 14626 et du manuscrit 986 de l'Arsenal qui appartint au collègue de Gaguin, Ambroise de Cambrai, à partir de 1488, avant qu'il ne le cède au collège de Navarre en 1496 (voir f. $199^{\mathrm{v}}$, mort de Louis VI sans copie de l'épitaphe). Il semble aussi que la mention de la fondation de l'établissement victorin par Louis VI (Compendium, f. 48v), vienne du Memoriale, f. 375 du ms. lat. 15011.

${ }^{52}$ Les vers ont été publiés en intégralité par H. Sauval, Histoire et antiquités de la ville de Paris, Paris, 1724, t. 1, livre IV, p. 409 et dans l'Épitaphier du vieux Paris, XII, éd. H. Verlet, Paris, $1999, \mathrm{n}^{\circ} 5789$ p. 352-3 ; les deux derniers vers manquants sont Christi centeno cum mille, decem et tribus anno, / Templum hoc victoris struxit regale honoris. On peut certes toujours imaginer que ces vers étaient mal lisibles et soutenir que la voie de leur connaissance
} 
emprunts sont certains comme celui du distique interdisant aux femmes l'accès à la chapelle St-Pharon de Meaux ${ }^{53}$ ou les ultima verba de Philippe le Bel à ses fils ${ }^{54}$.

Hormis les auteurs victorins, quelques manuscrits de la librairie présentent des configurations sinon exclusives du moins rares. Outre le Liber historiarum partium Orientis d'Hayton, le ms. lat. 14693 de la BNF contient l'Histoire romaine d'Eutrope interpolée et continuée, à partir du livre XI, par Paul Diacre, puis l'Historia Longobardorum de ce dernier. Gaguin utilise et l'Histoire romaine ${ }^{55}$ et l'Histoire des Lombards ${ }^{56}$, attribuant d'ailleurs à Eutrope un passage redevable à Paul Diacre dans le livre IV de l'Historia romana. Le manuscrit victorin lui permettait de puiser aux œuvres et de se méprendre comme il le fait. L'hypothèse de la consultation des deux auteurs dans le même manuscrit est renforcée par le fait qu'il les a utilisés l'un et l'autre seulement pour la dernière édition du Compendium, comme s'il avait découvert le volume après 1498. Hélas, parmi les manuscrits que l'éditeur de l' Historia Longobardorum énumère, un autre présente conjointement les deux œuvres de Paul Diacre : le lat. 6815 de la BNF, daté du XIV siècle et comprenant en plus Martin le Polonais ${ }^{57}$. Comment trancher? Et l'éventualité séduisante d'une découverte globale par Gaguin des trésors de Saint-Victor pour la dernière révision de son Histoire de France ne résiste pas à la trop forte probabilité d'emprunts accomplis dès la rédaction primitive de $1495^{58}$.

Il y a surtout ce vaste recueil fait pour Simon de Plumetot. Beaucoup de composantes de son contenu hétéroclite ont pu servir de sources à Robert Gaguin, voire à Nicole Gilles. La Geste des rois de France en français jusqu'à Philippe de Valois semble avoir fourni aux deux historiens l'origine du surnom d'Hugues Capet, ainsi nommé parce qu'il enlevait par espièglerie leur chaperon aux jeunes gens de sa compagnie $^{59}$, ce qu'une note marginale met en évidence aux yeux du lecteur pressé. L'Historia unde processit regnum Yvetotis apporte au Trinitaire comme au notaire et secrétaire du roi tout ou partie de la matière de leur développement sur le sujet ${ }^{60}$. Utilisés par nos auteurs, les ouvrages d'Eginhard et de Guillaume de Jumièges

fut bien épigraphique, comme le fait, à propos de l'épitaphe de Clovis à Sainte-Geneviève reproduite dans le Compendium, P. Bourgain, «Le poème sur Clovis attribué à saint Remi », Francia, 31-1 (2004), p. 141-149.

${ }^{53}$ Compendium, f. $20^{\mathrm{v}}$ / Memoriale historiarum, BNF ms. lat. 15011, f. $246^{\mathrm{v}}$.

${ }^{54}$ Compendium, f. $70^{\mathrm{V}}$ / Memoriale historiarum, BNF ms. lat. 15011, f. 483.

${ }^{55}$ Compendium, f. $1^{\mathrm{v}}$ et $167^{\mathrm{v}} /$ Eutrope, Breviarium ab urbe condita, livre IV (fondation de Pavie) puis livre XI (revenant à Paul Diacre), victoire de Valentinien sur les Saxons.

${ }^{56}$ Compendium, f. 26 et $26^{\mathrm{V}}$ / Paul Diacre, Historia Longobardorum, I, 2 ; II, 5,7 et 9; IV, 38.

${ }^{57}$ Édition due à G. Waitz et parue à Hanovre, 1878, p. 47.

${ }^{58}$ Cf. F. Collard, Un historien au travail..., p. 341.

${ }^{59}$ BNF ms. lat. 14663, f. 18 / Compendium, f. 42 ; Annales et Croniques de France, f. 79 : «dient aucunes croniques ».

${ }^{60}$ BNF ms. lat. 14663, f. 48 ; Compendium, f. 9 ; Annales et Croniques de France, f. 19; Gaguin a une hésitation lors du premier jet de son texte car il parle d'un pape Agapet ou Vigile, l'un et l'autre ayant régné en 536, date de l'épisode supposé de la mort de Gautier d'Yvetot; puis il se rallie à Agapet. Gilles tait le nom du pontife. Le Memoriale historiarum permettait aisément, dans certaines copies truffées de dates marginales du moins, de prendre ces repères pontificaux. 
figuraient aussi, avec cependant, pour le premier, l'appellation de Guertinus qu'on ne retrouve ni chez Gaguin (il ne dénomme pas l'auteur de la Vita Karoli, sans doute perplexe devant la variation des noms constatée d'ailleurs par le copiste du manuscrit $)^{61}$ ni chez Nicole Gilles qui reprend l'appellation d' «Eginaux $»^{62}$. Encore moins sûre est l'exploitation des passages copiés du prologue de la traduction française de la Cité de Dieu donné par Raoul de Presles car une édition parue en 1486 à Abbeville existait, que détenait d'ailleurs Nicole Gilles. Un autre doute surgit de l'existence d'un manuscrit en partie copié sur celui de Saint-Victor mais étranger aux pupîtres de l'abbaye: le ms. 1094 de l'Arsenal. Sa provenance en rend la consultation par Gilles plus vraisemblable que celle du volume de Saint-Victor parce qu'il appartint au sien collègue notaire et secrétaire du roi Jean Budé qui l'avait fait copier en décembre $1486^{63}$. Une bonne partie des textes contenus dans ce recueil proviennent indiscutablement du manuscrit de Saint-Victor : «Guertinus » (qu'une note marginale dit aussi appelé Bernardus $)^{64}$, Guillaume de Jumièges, les extraits de Raoul de Presles plus le poème sur les lys ${ }^{65}$, la chronique abrégée des rois de France en français avec l'origine du surnom «Capet ${ }^{66}$, l'Historia unde processit regnum Yvetotis $^{67}$, sans parler d'autres pièces qui ne nous intéressent pas directement. Même si l'hypothèse est plus incertaine, on ne peut écarter que Gaguin ait eu recours à ce manuscrit étant donné les rapports ayant pu exister entre le père de Guillaume Budé et l'humaniste trinitaire dont le Compendium fut traduit, en 1513-1514, par celui-là même à qui l'auteur du De Asse avait résigné sa charge de notaire et secrétaire du $\operatorname{roi}^{68}$.

Le gros recueil des actes des procès de Jeanne d'Arc pose aussi problème. A Gaguin qui donne des détails précis sur la personnalité et la condamnation de la

\footnotetext{
${ }^{61}$ En marge, f. 49, il a écrit que ce nom de Guertinus se trouvait dans un livre de Saint-Denis.

${ }^{62}$ Annales et Croniques de France, f. $45^{\mathrm{v}}$.

${ }^{63}$ M.-C. Garand, «Les copistes de Jean Budé (1430-1502) », Bulletin de l'IRHT, 15 (19671968), p. 293-331. Sur Budé, voir la notice de l'ouvrage d'A. Lapeyre et R. Scheurer, Les notaires et secrétaires du roi sous les règnes de Louis XI, Charles VIII et Louis XII (14611515), Paris, 2 vol., Bibliothèque nationale, 1978, t. 1 p. 70-1. C'est entre 1480 et 1490 qu'il se constitua une bibliothèque, faisant par exemple copier un texte sur les serpents dans un manuscrit du collège de Sorbonne (Ars, ms. 1094, f. 143). Une maladie contractée à ce moment-là le rendit curieux des livres de médecine qu'il fit copier en nombre, peut-être aussi à Saint-Victor dont les fonds, on l'a dit, étaient riches de ce genre d'ouvrages. Le manuscrit Ars. 1094 passa ensuite aux Minimes.

${ }^{64}$ Ars, ms. 1094, f. $7 \mathrm{v}$ : in libro sancti dionisii ponitur secundum bernardum et forte vocabatur auctor bernardus guertini

${ }^{65}$ Ibid., f. $137^{\mathrm{v}}$ et 142 .

${ }^{66}$ Ibid., f. $123^{\mathrm{v}}$.

${ }^{67}$ Ibid., f. 155.

${ }^{68}$ A. Lapeyre et R. Scheurer, Les notaires et secrétaires du roi, t. 1 p. 71 . Sur cette traduction, voir F. Collard, «Histoire de France en latin et histoire de France en langue vulgaire : la traduction du Compendium de origine et gestis Francorum de Robert Gaguin au début du $\mathrm{XVI}^{\mathrm{e}}$ siècle », Histoires de France, historiens de la France, Actes du colloque international de la Société de l'Histoire de France (Reims, mai 1993), éd. Ph. Contamine et Y.-M. Bercé, Paris, Champion, 1994, p. 91-118.
} 
Pucelle $^{69}$, il pouvait évidemment apporter tous les éléments utiles, comme il en apporta plus tard à Étienne Pasquier ${ }^{70}$. Mais en avait-il vraiment besoin, lui qui connaissait intimement François Ferrebouc, notaire pontifical qui rédigea les documents de $1456 ?^{71}$

Le potentiel historiographique de la bibliothèque de Saint-Victor ainsi que sa facilité d'accès en faisaient indéniablement une fontaine généreuse où puiser des données. Nos trois auteurs, même l'Italien, étaient loin des préventions de Rabelais contre ce temple de la culture scolastique. Par simple utilitarisme ou par attachement à l'établissement victorin, probable au moins chez le frère trinitaire Gaguin, ils pouvaient recourir aux volumes de sa «librairie » qui accueillit d'ailleurs les œuvres de chacun de nos trois auteurs ${ }^{72}$. Il est pourtant rien moins que certain qu'ils l'aient fait.

De sérieuses présomptions existent certes pour une utilisation de quelques manuscrits historiques victorins par Gaguin, mais il est audacieux de les élever au rang de certitudes comme le faisait Thuasne ${ }^{73}$. Elles diminuent avec Gilles et s'évanouissent avec Paul Émile. Peu disert sur ses sources, ce dernier disposait ex officio des collections royales. Son œuvre touffue ne laisse pas penser qu'il se servît du fonds victorin mais il ne faut y voir ni une incertaine défiance ni un improbable parti-pris, seulement de l'indifférence envers une ressource superflue. Nicole Gilles écrivait de l'histoire laïque. Cela ne signifie évidemment pas qu'il ait fait fi de l'histoire monastique ni de l'historiographie latine mais ses horizons culturels, à la différence de certains de ses prédécesseurs des années 1400 , ne le portaient pas vers Saint-Victor. Au mieux a-t-il eu indirectement recours aux richesses historiques de l'abbaye par l'intermédiaire d'un sien collègue notaire et secrétaire. Reste Gaguin, voisin et ami des chanoines, admirateur de la pensée victorine, en quête de sources diversifiées. Il a assurément connu le Memoriale historiarum de Jean de SaintVictor. Il a indéniablement recouru à des textes contenus dans des manuscrits victorins. Le problème est qu'il existait pour la plupart de ces textes des copies consultables ailleurs qu'au couvent. À bien y regarder, en l'état actuel des

${ }^{69}$ Compendium, f. 117 et 120.

${ }^{70} \mathrm{Ph}$. Contamine, «Naissance d'une historiographie... », p. 255.

${ }^{71}$ C'est à lui qu'il adresse la fameuse lettre de Burgos en 1468 (Epistole et orationes, t. 1 p. 185 et sq.)

${ }_{72}$ Voir le catalogue de la bibliothèque de Saint-Victor établi par frère Étienne Regnard en 1623 (BNF ms. lat. 15169, f. 78 et sq. cotes O 53, 54 (deux Gaguin en latin), 55 (Gaguin en français), 63 (Paul Emile), 65, 66 et P 74 (Nicole Gilles). L'auteur a commis une confusion très instructive sur les titres des ouvrages de Paul Emile et de Robert Gaguin en les intervertissant, signe de leur quasi équivalence dans l'esprit des lecteurs modernes.

${ }^{73}$ Epistole et orationes, t. 1 p. 123. 
recherches, aucun passage de son Compendium ne paraît absolument redevable à un volume de la librairie de Saint-Victor. Aucun sauf les vers en l'honneur du fondateur de l'abbaye à laquelle le Trinitaire rendait ainsi hommage, mais sans faire de sa librairie sa bibliothèque de référence, loin s'en faut.

Franck Collard Université de Paris Ouest Nanterre 